\title{
Analytical Results of the 1997 R-Reactor Disassembly Basin Sampling Program
}

by

J. B. Pickett

Westinghouse Savannah River Company

Savannah River Site

Aiken, South Carolina 29808

DOE Contract No. DE-AC09-96SR18500

This paper was prepared in connection with work done under the above contract number with the U. S. Department of Energy. By acceptance of this paper, the publisher and/or recipient acknowledges the U.S. Government's right to retain a nonexclusive, royalty-free license in and to any copyright covering this paper, along with the right to reproduce and to authorize others to reproduce all or part of the copyrighted paper.

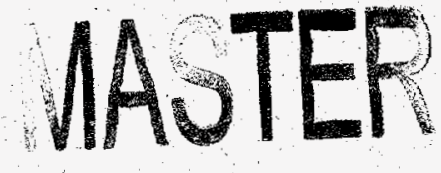




\section{DISCLAIMER}

This report was prepared as an account of work sponsored by an agency of the United States Government. Neither the United States Government nor any agency thereof, nor any of their employees, makes any warranty, express or implied, or assumes any legal liability or responsibility for the accuracy, completeness, or usefulness of any information, apparatus, product, or process disclosed, or represents that its use would not infringe privately owned rights. Reference herein to any specific: commercial product, process, or service by trade name, trademark, manufacturer, or otherwise does not necessarily constitute or imply its endorsement, recommendation, or favoring by the United States Government or any agency thereof. The views and opinions of authors expressed herein do not necessarily state or reflect those of the United States Government or any agency thereof.

This report has been reproduced directly from the best available copy.

Available to DOE and DOE contractors from the Office of Scientific and Technical Information, P.O. Box 62, Oak Ridge, TN 37831; prices available from (615) 576-8401.

Available to the public from the National Technical Information Service, U.S. Department of Commerce, 5285 Port Royal Road, Springfield, VA 22161. 


\section{DISCLAIMER}

Portions of this document may be illegible electronic image products. Images are produced from the best available original document. 
FDD-ENG-98-0029

\section{ANALYTICAL RESULTS OF THE 1997 R-REACTOR DISASSEMBLY BASIN} SAMPLING PROGRAM

Prepared by J. B. Pickett

Facilities Decommissioning Division (FDD)

Engineering Department 
FDD Engineering

R-Disassembly Basin

Analytical Results on 1997 Sampling Program

FDD-ENG-98-0029

$4 / 1 / 98$

Page 1 of 14

ANALYTICAL RESULTS OF THE 1997 R-REACTOR DISASSEMBLY BASIN SAMPLING PROGRAM

Facilities Decommissioning Division, FDD Engineering Department

Originator

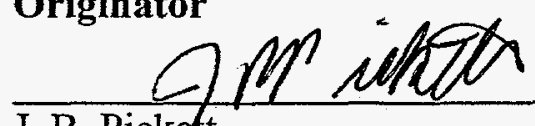

Date $4 / 16 / 98$

Title: Fellow Scientist, FDD Engineering

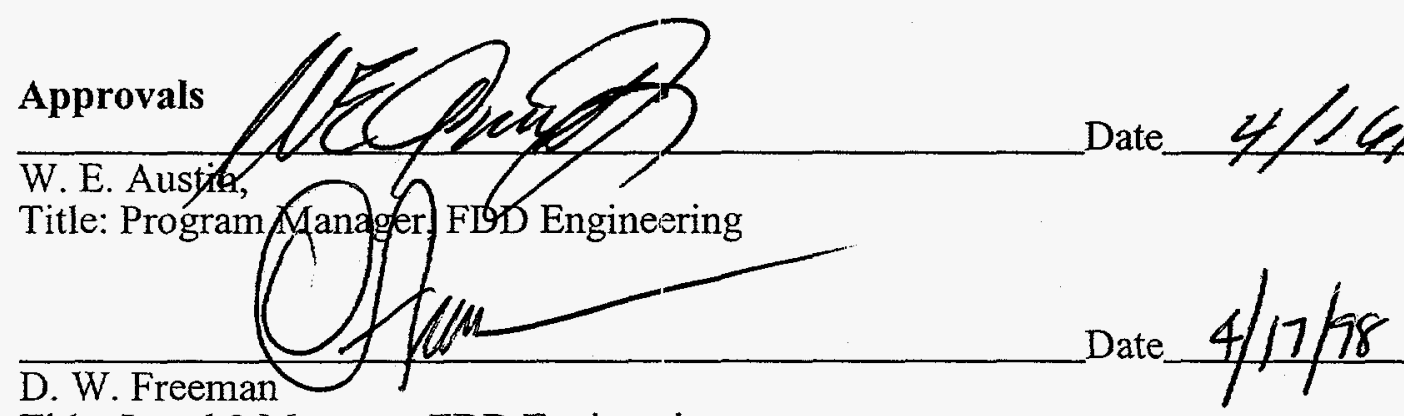

Title: Level 2 Manager, FDD Engineering 


\section{R-REACTOR DISASSEMBLY BASIN ANALYSICAL SAMPLING RESULTS}

\section{OBJECTIVE}

The primary objective of this document (FDD-ENG-98-0029) is to provide the analytical results of the sampling program conducted in 1997 in the R-Reactor Disassembly Basin. The key conclusions on the analytical results are summarized, and the current results are compared to previous sampling campaign results. One of the key objectives of this program was to conclusively define whether the sediment layer at the bottom of the basin is a PCB/TSCA waste (i.e., a material containing greater than $50 \mathrm{mg} / \mathrm{km}$ Polychlorinated Biphenyl compounds). It was determined that the sediment layer is NOT a PCB hazardous waste.

The radiation survey conducted as a part of this program and the sampling techniques and sampling locations sampled during this study are described in a companion report (FDD-ENG98-0019).

\section{BACKGROUND}

Fuel and target assemblies were discharged from R-Reactor into the water filled disassembly basin. The reactor assemblies were initially stored in the Vertical Tube Storage (VTS), or Temporary Tube Storage, where the short-lived radionuclides decayed. The assemblies were then disassembled in the "Machine Basin" (MB), with the disassembled pieces stored in the "Bucket Storage" (BKS) and/or "Dry Cave Section (DCS) areas. Two other smaller areas, the "Monitor Pin Basin" (MPB) and the "Transfer Bay" (TB) are also included in the R-Reactor Disassembly Basin. The Emergency Disassembly Basin (EB) was the location of a 1957 experiment involving a fuel assembly inside a calorimeter, which ruptured during testing. In 1964 the EB was drained of water, backfilled with clay, and topped with a concrete cap.

The sampling conducted in this campaign determined that there is a sediment layer at the bottom of the basin which is $\sim 1 / 4$ to $3 / 8$ inch deep. The sediment is approximately $6 \mathrm{wt}$. $\%$ solids and is composed primarily of fine particles of iron hydroxide, or iron oxide (rust), aluminum hydroxide, and sand.

\section{Previous Sampling Study Results}

The previous sampling campaigns in 1994 and 1996 were conducted to meet the objective of the Reactor Division Disassembly Management Plan, which was to transition the reactor basin to a Safe Storage Condition. The sediment and the water in the basin were sampled and analyzed in three previous sampling episodes, and the results were summarized in Ref. 1. Two composite samples were measured in 1994 by an off-site laboratory for a full suite of inorganic and organic constituents. The only constituent of concern detected in those programs was Aroclor 1254, a Polychlorinated Biphenyl (PCB) compound, at concentrations of 15.2 and $99.2 \mathrm{mg} \mathrm{PCB} / \mathrm{kg}$ of sediment. The $99.2 \mathrm{mg} / \mathrm{kg}(\mathrm{ppm})$ analysis exceeded the PCB regulatory limit of $50 \mathrm{ppm}$.

\section{ANALYSIS SUMMARY}

\section{Chemical and Radionuclide Analytical Results Summary}

The 1997 analytical results indicate that the sediment is not a PCB waste. Aroclor 1260 was detected at a mean concentration of $1.87 \mathrm{ppm}$, well below the regulatory limit of $50 \mathrm{ppm}$. A statistical analysis of the PCB concentration data indicates that the PCB concentration in the sediment is less than $2.86 \mathrm{ppm}$, at a $99 \%$ confidence level (Table 7). This is far below the limit of $50 \mathrm{ppm}$. 
A thin film was observed to be present on the surface of the basin liquid. The film is estimated to be $\sim 1 / 16$ to $1 / 8 "$ thick. The film itself is $\sim 1000 \mathrm{ppm} w t . \%$ solids, and is believed to be primarily calcium carbonate, with some oil and grease $(400-500 \mathrm{ppm})$. Since the majority of the $\sim 5,000,000$ gallons of liquid is supersaturated with calcium (vs. precipitation of calcium carbonate) the film is believed to be similar to a hard water "bath tub ring", i.e., calcium and magnesium carbonates, with some low density oils and greases. One PCB (Aroclor 1260) was detected in the film portion, at less than $0.07 \mathrm{ppm}$; Table 2.

The primary chemical constituents present in the liquid portion are $\mathrm{Ca}, \mathrm{K}$, and $\mathrm{Na}$ hydroxides, at an almost neutral $\mathrm{pH}$ of 7.6. $\mathrm{PCB}$ concentrations in the liquid portion were determined to be less than $0.1 \mu \mathrm{g} / \mathrm{L}(\mathrm{ppb})$.

The primary radionuclides detected in the film and liquid portions were Cs-137 ( $\sim 90,000$ $\mathrm{pCi} / \mathrm{L})$, Sr-90, $(\sim 25,000 \mathrm{pCi} / \mathrm{L})$, and tritium $(\sim 38,000 \mathrm{pCi} / \mathrm{ml})$. Carbon 14 was detected at a $25-$ $200 \mathrm{pCi} / \mathrm{L}$ level, while all other radionuclides were less that $50-100 \mathrm{pCi} / \mathrm{L}$. The film and liquid radionuclide data is given in Tables 3 and 5.

The primary radionuclides detected in the sediment portion were: $\mathrm{Cs}-137(21,000 \mathrm{pCi} / \mathrm{gm}), \mathrm{Sr}-$ 90 (3,000 pCi/gm), Co-60 (48,000 pCi/gm), Pu-241 (22,600 pCi/gm), C-14 (6,000 pCi/gm), and tritium $(397,000 \mathrm{pCi} / \mathrm{gm})$, The sediment radionuclide data is given in Table 8 .

The primary chemical constituents in the sediment are $\mathrm{Al}, \mathrm{Fe}, \mathrm{Ca}, \mathrm{Mn}, \mathrm{Zn}$, and silica. At the sediment $\mathrm{pH}$ of 8.1 , the metals are believed to be primarily present as hydroxides, although the visual appearance of the sediment (a dark reddish brown), indicates that the Fe is probably present as ferrous or ferric oxide.

There were no major differences between the current (1997) results and those conducted by SRTC and Rust Engineering Lab. in 1994, with two exceptions; the Cs-137 and Co-60 results in the sediment were approximately 10x higher in the 1997 study vs. the 1994 SRTC results.

\section{DISCUSSION}

\section{Surface Film}

The surface film was sampled by drawing an open mouth container across the surface of the water. This obtained mostly the liquid below the film, and only a slight amount of film. The film chemical and radionuclide analyses of film and liquid are very similar. Tables 1 and 3 compare the film's liquid chemical and radionuclide analytical results.

Only one of the four film samples that was collected contained a significant amount of film material (VTS-1; Table 2). This is evidenced by the higher total wt. \% solids (194 ppm) vs. the other film samples and the liquid samples; $145 \mathrm{ppm}$. The VTS-1 sample was also the only film sample to have any measured suspended solids ( $\sim 72 \mathrm{ppm})$.

Based on the results for VTS-1, it was calculated that the film comprised approximately $7 \%$ of the overall VTS-1 sample. The oil and grease was then calculated to be $\sim 500 \mathrm{ppm}$ in the film portion itself (Table 2). The only chemical constituent that was elevated in the VTS-1 sample (vs. the other film samples) was calcium. Calcium was calculated to be approximately $660 \mathrm{mg} / \mathrm{L}$ in the film vs. $12-13 \mathrm{mg} / \mathrm{L}$ in the liquid.

The calcium is believed to be present as calcium carbonate, since the concentration of $\mathrm{Ca}$ in the basin liquid exceeds the solubility product $\left(\mathrm{k}_{\mathrm{sp}}\right)$ of $\mathrm{CaCO}_{3}$. The $\mathrm{k}_{\mathrm{sp}}$ of $\mathrm{CaCO}_{3}$ is $9 \times 10^{-9}$, and the $\mathrm{Ca}$ concentration needed to precipitate $\mathrm{CaCO}_{3}$ is $3.8 \mathrm{mg} / \mathrm{L}$. The calcium in the liquid is $12-13$ $\mathrm{mg} / \mathrm{L}$, so the liquid is super-saturated with respect to $\mathrm{CaCO}_{3}$ precipitation. 
This explains the field observation that the film appeared to be thicker, or denser (more opaque) at the conclusion of the sampling program vs. the beginning. The agitation of the radiation survey instrument and submersible pump probably created small crystals, or dispersed small crystals into the liquid, which initiated precipitation of the carbonate. This can be expected to occur during the eventual treatment of the liquid or sediment, and should be considered when designing the filtration equipment. Sodium carbonate or bicarbonate could be added to the liquid prior to the initial roughing filtration, which should remove most of the calcium and magnesium. This would prevent fouling of the subsequent Sr-90 and Cs-137 removal systems.

\section{Liquid Portion}

The chemical and radionuclide results for the liquid portion in the Disassembly Basin are given in Tables 4 and 5. The current, 1997 analyses are compared to the results of the 1994 sampling program. The only significant difference is that Sr-90 was detected in the 1997 campaign, whereas it was not requested to be analyzed in the 1994 program. The chemical analysis conducted by Clemson Technical Center (CTC) - Rust Engineering Lab., on one sample of liquid in 1994 are quite different in most constituents from the 1997 results. Since the 1994 SRTC and 1997 GEL results agree well on most chemical constituents, the Rust results will not be further utilized.

\section{Sediment Results}

The sediment results are believed to be a fairly accurate representation of the layer at the bottom of the basin, due to the setting characteristics of the material. The sediment settled very quickly in the sample collection carboys (usually overnight), resulting in a dense layer and a clear supernate. As described in the companion report, 50 to 60 gallons of sediment/water mixture were collected, which was then allowed to settle out - in some cases for 1 to 2 weeks. The resulting 4 to 6 inch deep sediment layer in the collection bottle (15 gallon carboy) was fairly homogeneous, as observed during the transfer of the sediment to the laboratory sample bottles. The weight $\%$ solids $(\sim 6 \%)$ in the R-Basin sediment is also similar to that measured in the LBasin Settler tank, and is what might be expected of a hydroxide precipitate in contact with a large volume of liquid.

The chemical and radionuclide results (Tables $6 \& 8$ ) from the 1997 study agree reasonably well with the 1994 results, except for Cs-137 and Co-60. Since the GEL analyses had significantly lower detection limits (pCi/gm) than the 1994 SRTC analyses $(\mu \mathrm{Ci} / \mathrm{gm})$, the higher GEL values are preferred.

One of the main objectives of this study was to determine if the sediment layer contained significant concentration of PCB's. The PCB analyses are given in Table 7, and are quite consistent. The standard deviation for the 11 composites, and one split sample, is $1.26 \mathrm{mg} / \mathrm{Kg}$ (ppm). The upper limit for the PCB concentration is 2.86 , at a $99 \%$ confidence level. This is based on a "one-tailed t-statistic" probability calculation (Ref. 2), given below:

The upper limit for the PCB concentration = mean $+t(0.99, n-1) s / n^{1 / 2}$. The $t$ statistic at the $99^{\text {th }}$ percentile for $12-1=11$ values; is 2.718 . The mean is 1.87 , the standard deviation (s) is $1.26, \mathrm{n}$ $=12$.

Therefore, $1.87 \mathrm{ppm}+(2.718 \times 1.26 / 3.464)=1.87+0.99=2.86 \mathrm{ppm}$.

This $99^{\text {th }} \%$ value of $2.86 \mathrm{ppm}$ is far below the regulatory limit of $50 \mathrm{ppm}$, so it is concluded that the R-Basin sediment is not a PCB/TSCA waste. 
$\underline{\text { Radionuclide Summary }}$

\begin{tabular}{|c|c|c|c|}
\hline Radionuclide & Curies in Liquid & Curies in Sediment & Total C \\
\hline Tritium & 714 & 0.45 & 714 \\
\hline Sr-90 & 0.44 & 0.004 & 0.44 \\
\hline Cs-137 & 1.74 & 0.02 & 1.76 \\
\hline Co-60 & - & 0.05 & 0.05 \\
\hline
\end{tabular}

Where $\mathrm{T}^{3}$ in liquid $=38,000 \mathrm{pCi} / \mathrm{L} \times 5,000,000$ gallons liquid $\times 3.75 \mathrm{~L} / \mathrm{Gal}$., and $10^{12} \mathrm{pCi} / \mathrm{Ci}$

$\mathrm{T}^{3}$ in sediment $=397.000 \mathrm{pCi} / \mathrm{gm} \times 5,000$ gallons sediment $\times 3.75 \mathrm{~L} / \mathrm{Gal} . \times 6 \%$ solids. $10^{12} \mathrm{pCi} / \mathrm{Ci}$

\section{Definitions and Acronyms}

Sediment For this study, the sediment is defined as the $\sim 1 / 4$ to $3 / 8$ inch deep layer of material at the bottom of the Disassembly Basin. The analyses indicated $\sim 5-10 \%$ total solids.

Supernate This is defined as the liquid in direct contact with the sediment solids, and that liquid which can be separated from the sediment by filtration or decanting.

Basin Liquid The majority of the liquid in the basin, above the sediment layer to the surface.

Basin Surface This top layer of liquid in the basin, which has an "oil sheen" and a surface film. The film layer is $\sim 1 / 32$ to $\sim 1 / 8$ inch thick. The surface layer was sampled to a depth of 2-3 inches.

BOD Biological Oxygen Demand

BETX Benzene, Ethylbenzene, Toluene, and Xylene

COD Chemical Oxygen Demand

CTC Clemson Technical Center, Rust Engineering Laboratory, Clemson, SC

EPA U. S. Environmental Protection Agency

GEL General Engineering Laboratory, Charleston, SC

PCB's Polychlorinated Biphenyl compounds

PPE Personal Protective Equiprnent

PVC Polyvinyl Chloride

TDS Total Dissolved Solids

TPH Total Petroleum Hydrocarbons

TSS Total Suspended Solids

SRTC Savannah River Technology Center

TSCA Toxic Substances Control Act

\section{Sample Areas}

VTS Vertical Tube Storage

MB Machine Basin

BKS Bucket Storage Section

DCS Dry Cave Section

MPB Monitor Pin Basin

TB Transfer Basin 


\section{REFERENCES}

1. J. B. Pickett, "R-Reactor Disassernbly Basin Radiation Survey and Sampling Plan", FDDENG-97-0044, Rev. 1, August 13, 1997 (Rev. 1 is dated 4/8/98).

2. U. S. Environmental Protection Agency, "Statistical Analysis of Groundwater Monitoring Data at RCRA Facilities, Interim Final Guidance", PB-89151047, February, 1989, Washington, DC.

\section{ATTACHMENTS}

1 J. D. C. Crawford to B. Nora, “Job $97047 F$ (50 Day TAT)", January 30, 1998. GEL Laboratory results (metals and radionuclides) for film samples collected $8 / 27 / 97$.

2. J. D. C. Crawford to J. B. Pickett, "Job 97344 - Attached are the results from General Engineering Laboratory for Job 97344", February 5,1998. GEL Laboratory results (organics and anions) for film samples collected 12/03/97.

3 J. D. C. Crawford to B. Nora, "Job 97293F", February 20, 1998. GEL Laboratory results (metals, organics, and radionuclides) for liquid samples collected 12/08/97.

4 J. D. C. Crawford to J. B. Pickett, "Job 97428 F - Attached are the results from General Engineering Laboratory for Job 97428F", March 31,1998. GEL Laboratory results (metals, organics, and radionuclides) for sediment samples collected $1 / 14 / 98$.

Note, the Attachments are only attached to the author's copy and the FDD/TIS file copies 
1997 R-DISASSEMBLY ANALYTICAL RESULTS: SURFACE LAYER, METALS

\author{
Sample Location \\ Sample ID \\ Laboratory \\ Lab. ID \\ Date Sampled \\ $\%$ Solids, $\mathrm{mg} / \mathrm{L}$ (ppm) \\ $\%$ Film in Sample*
}

\section{Total Metals}

Aluminum

Antimony

Arsenic

Barium

Beryllium

Boron

Cadmium

Calcium

Calcium, in film ${ }^{* *}$

Cesium

Chromium

Cobalt

Copper

Iron

Lead

Magnesium

Manganese

Molydenum

Nickel

Potassium

Selenium

Silver

Sodium

Strontium

Thallium

Tin

Titanium

Uranium

Vandium

Zinc
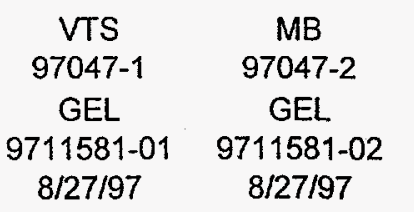
DCS
97047-3
GEL
9711581-03
$8 / 27 / 97$

164
0.0018

147
0.0001

143
Neg.'

Total Constituent Concentration, $\mu \mathrm{g} / \mathrm{L}$ (ppb)

\begin{tabular}{cc|c|}
$\begin{array}{c}\text { BKS/MPB } \\
97047-4 \\
\text { GEL }\end{array}$ & $\begin{array}{c}\text { Average, } \\
\text { Surface }\end{array}$ & $\begin{array}{c}\text { Average. } \\
\text { Liquid }\end{array}$ \\
\cline { 2 - 2 } $\begin{array}{c}\text { G711581-04 } \\
8 / 27 / 97\end{array}$ & GEL & $\begin{array}{c}\text { GEL } \\
\text { (Table 4) } \\
12 / 97\end{array}$ \\
143 & $8 / 97$ & \\
Neg.' & 149 & 146
\end{tabular}

VTS=Vertical Tube Storage Basin; MB = Machine Basin; DCS = Dry Cave Section;

BKS/MPB = Open area between Bucket Storage Section and Monitor Pin Basin

\% Film in sample; assume film is $1000 \mathrm{mg} / \mathrm{L}$ total solids (same as Table 3),

and average \% solids in liquid portion is $146 \mathrm{mg} / \mathrm{L}$ (Table 4)

Then, for the VTS, $164 \mathrm{mg} /-146 \mathrm{mg} / \mathrm{L}=18 \mathrm{mg} / \mathrm{L} \mathrm{from} \mathrm{the} \mathrm{film,} \mathrm{at} 1000 \mathrm{mg} / \mathrm{L}$, this equates to $18 \mathrm{ml}$ in one Liter, or $0.0018 \%$

${ }^{* \star} \mathrm{Ca}, \mathrm{mg} / \mathrm{L}$ in film: The difference between the VTS Ca concentration $(13.5 \mathrm{mg} / \mathrm{L})$ and the average liquid $\mathrm{Ca}(12.3 \mathrm{mg} / \mathrm{L})$ is

$1.2 \mathrm{mg} / \mathrm{L}$. Therefore, $18 \mathrm{ml}$ (in 1 liter of sample) accounts for $1.2 \mathrm{mg}$ of $\mathrm{Ca}$, or

$0.0018 \mathrm{~L}{ }^{*} \times \mathrm{mg} / \mathrm{L} \mathrm{Ca}=1.2 \mathrm{mg} \mathrm{Ca} ;=1.2 \mathrm{mg} \mathrm{Ca} / 0.0018 \mathrm{~L}=\sim 660 \mathrm{mg} / \mathrm{L} \mathrm{Ca}$ in the film 
SURFACE LAYER; ORGANICSIPCB'S/ANIONS

Sample Location

Sample ID

Laboratory

Lab. ID

Date Sampled

Total wt \% Solids (Measured)

Total Dissolved Solids (TDS)

Total Suspended Solids (TSS)

Wt \% Film in Sample (Calculated) ${ }^{*}$

Total wt \% Solids; (Calcuated ${ }^{\star \star}$ )

$\mathrm{pH}$

Oil and Grease (measured)

Oil and Grease (calculated in film portion)

Total Organic Carbon (TOC)

Total Organic Carbon (calculated in film portion)

Biological Oxygen Demand (BOD)

Chemical Oxygen Demand (COD)

$\begin{array}{ccc}\text { VTS } & \text { MB } & \text { DCS } \\ 97344-4 & 97344-2 & 97344-1 \\ \text { GEL } & \text { GEL } & \text { GEL } \\ 9712179-04 & 9712179-02 & 9712179-01 \\ 12 / 3 / 97 & 12 / 3 / 97 & 12 / 3 / 97\end{array}$

BKS/MPB

97344-3

GEL

9712179-03

$12 / 3 / 97$

145

$\mathrm{mg} / \mathrm{L}$

151

142

138

$\mathrm{mg} / \mathrm{L} \quad 143$

145

144

138

$\mathrm{mg} / \mathrm{L} \quad 71.6$

4.8

2.4

5.4

$\% \quad 7.2$

$\mathrm{mg} / \mathrm{L} \quad 204$

0.48

0.24

0.54

150

146

143

7.84

8.23

8.20

$\mathrm{mg} / \mathrm{L} \quad 30.8$

$\mathrm{mg} / \mathrm{L} \quad 428$

$\mathrm{mg} / \mathrm{L} \quad 1.35$

$\mathrm{mg} / \mathrm{L} \quad 19$

0.73

152

0.14

1.47
613

$\begin{aligned} & 613 \\ < & 0.145\end{aligned}$

3.4

630

29

-

0.13

$\mathrm{mg} / \mathrm{L} \quad 7.0$

$\mathrm{mg} / \mathrm{L} \quad 42.7$

6.0

8.0

6.0

6.0

5.3

Volatile Organics

\begin{tabular}{|c|c|c|c|c|c|}
\hline $\begin{array}{l}\text { Diesel Range } \\
\text { Gasoline Range } \\
\text { BETX (ppb) }\end{array}$ & $\begin{array}{l}\mathrm{mg} / \mathrm{L} \\
\mu \mathrm{g} / \mathrm{L}\end{array}$ & $\begin{array}{l}0.19 \\
<100\end{array}$ & $\begin{array}{l}0.021 \\
<100\end{array}$ & $\begin{array}{l}0.015 \\
<100\end{array}$ & $\begin{array}{l}0.016 \\
<100\end{array}$ \\
\hline Benzene & $\mu \mathrm{g} / \mathrm{L}$ & $<1$ & $<1$ & $<1$ & $<1$ \\
\hline Toluene & $\mu \mathrm{g} / \mathrm{L}$ & $<1$ & $<1$ & $<1$ & $<1$ \\
\hline Ethyl-Benzene & $\mu \mathrm{g} / \mathrm{L}$ & $<1$ & $<1$ & $<1$ & $<1$ \\
\hline Xylenes & $\mu \mathrm{g} / \mathrm{L}$ & $<1$ & $<1$ & $<1$ & $<1$ \\
\hline \multicolumn{6}{|l|}{ Extractable Organics (ppb) } \\
\hline PCB-1016 & $\mu \mathrm{g} / \mathrm{L}$ & $<0.1$ & $<0.1$ & $<0.1$ & $<0.1$ \\
\hline PCB-1221 & $\mu g / L$ & $<0.1$ & $<0.1$ & $<0.1$ & $<0.1$ \\
\hline PCB-1232 & $\mu \mathrm{g} / \mathrm{L}$ & $<0.1$ & $<0.1$ & $<0.1$ & $<0.1$ \\
\hline PCB-1242 & $\mu \mathrm{g} / \mathrm{L}$ & $<0.1$ & $<0.1$ & $<0.1$ & $<0.1$ \\
\hline PCB-1248 & $\mu g / L$ & $<0.1$ & $<0.1$ & $<0.1$ & $<0.1$ \\
\hline PCB-1254 & $\mu g / L$ & $<0.1$ & $<0.1$ & $<0.1$ & $<0.1$ \\
\hline PCB-1260 & $\mu \mathrm{g} / \mathrm{L}$ & 1.98 & 0.088 & 0.17 & 0.058 \\
\hline PCB-1260 (calculated in film portion) & $\mu \mathrm{g} / \mathrm{kg}$ & 28 & 18 & 71 & 11 \\
\hline Bromide & $\mathrm{mg} / \mathrm{L}$ & 28.2 & 27.8 & 27.4 & 27.5 \\
\hline Chloride & $\mathrm{mg} / \mathrm{L}$ & 12.7 & 12.7 & 12.3 & 12.6 \\
\hline Fluoride & $\mathrm{mg} / \mathrm{L}$ & $<0.035$ & $<0.035$ & $<0.035$ & $<0.035$ \\
\hline Nitrite (as N) & $\mathrm{mg} / \mathrm{L}$ & $<0.01$ & $<0.01$ & $<0.01$ & $<0.01$ \\
\hline Nitrate (as N) & $\mathrm{mg} / \mathrm{L}$ & 0.46 & 0.43 & 0.42 & 0.43 \\
\hline SO4 & $\mathrm{mg} / \mathrm{L}$ & 3.19 & 3.29 & 3.06 & 3.14 \\
\hline Total PO4 & $\mathrm{mg} / \mathrm{L}$ & $<0.02$ & 0.05 & $<0.023$ & 0.10 \\
\hline
\end{tabular}

VTS =Vertical Tube Storage Basin; MB = Machine Basin; DCS = Dry Cave Section;

BKS/MPB = Open area between Bucket Storage Section and Monitor Pin Basin

"Wt \% Film Calculation:

ASSUME film wt. \% solids is $1000 \mathrm{mg} / \mathrm{L}$, or $1 \mathrm{mg} / \mathrm{mL}(0.1 \mathrm{Wt} \%)$ solids

Example; for the VTS sample, where TSS $=71.6 \mathrm{mg} / \mathrm{L}$, TDS $=143 \mathrm{mg} / \mathrm{L}$, and Total solids $=194 \mathrm{mg} / \mathrm{L}$, and if all the suspended solids are associated with the film.

**Then the calculated total Wt. \% solids $=x \mathrm{ml}$ film $\times 1 \mathrm{mg} / \mathrm{ml}=71.6 \mathrm{mg}$; Film volume $=\sim 72 \mathrm{mls}$ (in 1 liter) Then, $928 \mathrm{ml} \times 0.143 \mathrm{mg} / \mathrm{ml}=132 \mathrm{mgs}$ in liquid portion

$72 \mathrm{mgs}$ in film $+132 \mathrm{mgs}$ in liquid $=204 \mathrm{mgs} / \mathrm{L}$. total solids calculated, vs. $194 \mathrm{mg} / \mathrm{L}$ total measured 
TABLE 3

SURFACE LAYER - RADIONUCLIDES

age 9 of 14

Note, data analysis indicates the "surface sample" was primarily liquid, with very litle surface film

Sample Location

Sample ID

Laboratory

Lab. ID

Date Sampled

Gross Alpha

Non-volatile Gross Beta
VTS

GEL

9711581-05

$8 / 27 / 97$

9711581-06

$<5.16$

109,000
GEL

$8 / 27 / 97$

DCS

GEL

9711581-07

$8 / 27 / 97$

$<4.94$

95500

$<3.12$

91600
BKS/MPB
970474 GEL

9711581-08 $8 / 27 / 97$

109000

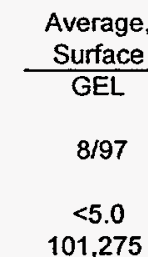

Average,

Liquid

(Table 5)

$12 / 97$

171

56,125

Alpha Spectroscopy, Nuclides Detected

U-233/234

$\mathrm{U}-238$

Pu-239/240

$\mathrm{pCi} / \mathrm{L}$

$\mathrm{PCi} / \mathrm{L}$

0.83

0.65

0.51
0.67

0.04

0.53
0.54

0.38

0.05

* < [ Det. Lim.

0.07

*

0.66

0.51

1.7

0.63

0.59

1.02

1.07

Gamma PHA Scan - Radionuclides Detected

\begin{tabular}{|c|c|c|c|c|c|c|c|}
\hline Cesium 137 & $\mathrm{pCi} / \mathrm{L}$ & 92900 & 90300 & 91800 & 92400 & 91,850 & 92,800 \\
\hline Other Radionucl & ected & & & & & & \\
\hline Strontium 90 & $\mathrm{pCi} / \mathrm{L}$ & 24800 & 26700 & 23700 & 27900 & 25,775 & 23,500 \\
\hline Tritium & pCi/ml & 37900 & 36300 & 36200 & 37100 & 36,875 & 38,075 \\
\hline Alpha Spectrosc & dides Be & Analytic & Detection Lir & & & & \\
\hline U-235 & $\mathrm{pCi} / \mathrm{L}$ & 0.06 & 0.08 & 0.03 & 0.1 & 0.07 & 10 \\
\hline $\mathrm{Np}-237$ & $\mathrm{pCi} / \mathrm{L}$ & 7.11 & 8.2 & $<11.3$ & 5.61 & 7.0 & 0.53 \\
\hline Pu-241 & pCill & 7.7 & 16.9 & 10.1 & 10.7 & 11 & - \\
\hline Pu-242 & $\mathrm{pCi} / \mathrm{L}$ & 0.3 & 0.16 & 0.17 & 0.05 & 0.17 & 0.18 \\
\hline Gamma PHA Sce & onuclide & low Anal & ical Lletectio & Limit & & & \\
\hline Actinium 228 & $\mathrm{pCi} / \mathrm{L}$ & 1.77 & * 8.56 & * 14.9 & $<17$ & 8.4 & 3.5 \\
\hline Antimony 124 & $\mathrm{pCi} / \mathrm{L}$ & $<143$ & 18.3 & 22.2 & 5.11 & 15 & - \\
\hline Antimony 125 & $\mathrm{pCi} / \mathrm{L}$ & 1.97 & $<178$ & 78.2 & 46 & 42 & - \\
\hline Barium 133 & $\mathrm{pCi} / \mathrm{L}$ & 17 & $<64$ & $<59$ & 40 & - & 3.9 \\
\hline Californium 249 & $\mathrm{pCi} / \mathrm{L}$ & 61.4 & $<63$ & $<57$ & 20 & * & - \\
\hline Californium 251 & $\mathrm{pCi} / \mathrm{L}$ & $<163$ & $<153$ & $<143$ & $<155$ & - & - \\
\hline Cerium 141 & pCi/l. & $<981$ & $<872$ & $<827$ & 9.8 & * & 14.9 \\
\hline Cerium 144 & $p$ Cill & $<367$ & 157 & $<298$ & $<357$ & - & - \\
\hline Cesium 134 & $\mathrm{pCi} / \mathrm{L}$ & $<35$ & 6.5 & 0.67 & 1.7 & 3.0 & - \\
\hline Cesium 135 & $\mathrm{pCi} / \mathrm{L}$ & $<203$ & 61 & $<188$ & $<195$ & - & - \\
\hline Cobalt 57 & $\mathrm{pCi} / \mathrm{L}$ & $<48$ & $<39$ & 18.8 & $<47$ & - & - \\
\hline Cobalt 58 & $\mathrm{pCi} / \mathrm{L}$ & 0.88 & 2.9 & 3 & $<18$ & 2.3 & 0.15 \\
\hline Cobalt 60 & pCill & 6.67 & 1.2 & 0.89 & 5.44 & 3.6 & 2.7 \\
\hline Europium 152 & $\mathrm{pCi} / \mathrm{L}$ & 29.5 & 5.2 & $<129$ & 13 & 16 & - \\
\hline Europium 154 & $\mathrm{pCi} / \mathrm{L}$ & 1.3 & $<13$ & 0.45 & 0.4 & 0.72 & - \\
\hline Europium 155 & $\mathrm{pCi} / \mathrm{L}$ & 21.4 & 5 & $<106$ & $<152$ & - & - \\
\hline Lead 212 & pCi/L & $<38$ & $<81$ & 36.6 & 30 & - & 9.4 \\
\hline Manganese 54 & $\mathrm{pCi} / \mathrm{L}$ & 0.91 & $<7.2$ & $<6.7$ & 1.4 & - & - \\
\hline Neptunium 239 & pCill & 9.89 & 91 & 32.8 & $<262$ & 45 & - \\
\hline Niobium 94 & $\mathrm{pCi} / \mathrm{L}$ & 1.41 & 0.2 & 4.1 & $<5.8$ & 1.9 & - \\
\hline Potasium 40 & $\mathrm{pCi} / \mathrm{L}$ & 21.3 & 72 & 35.4 & 0.5 & 32 & 48 \\
\hline Promethium 144 & $\mathrm{pCi} / \mathrm{L}$ & 1.23 & $<8.6$ & $<7.4$ & $<8.1$ & - & - \\
\hline Promethium 146 & $\mathrm{pCi} / \mathrm{L}$ & 39.8 & 43 & 9 & 0.09 & 23 & - \\
\hline Ruthenium 103 & $\mathrm{pCi} / \mathrm{L}$ & 40 & $<485$ & 44 & $<471$ & - & - \\
\hline Ruthenium 106 & pCi/L & 104 & 23 & 48 & $<394$ & 58 & 37 \\
\hline Sodium 22 & $\mathrm{pCi} / \mathrm{L}$ & 0.5 & $<5$ & 0.19 & 0.14 & 0.28 & - \\
\hline Tin 113 & $\mathrm{pCi} / \mathrm{L}$ & 9.91 & 6.5 & 67 & 23 & 27 & - \\
\hline $\operatorname{Tin} 126$ & $\mathrm{pCi} / \mathrm{L}$ & 1.5 & 0.6 & $<5.8$ & 1.2 & 1.1 & 1.5 \\
\hline Yttrium 88 & $\mathrm{pCi} / \mathrm{L}$ & $<8.33$ & 0.7 & 1.9 & $<8.7$ & - & 0.8 \\
\hline Zine 65 & $\mathrm{pCi} / \mathrm{L}$ & $<10.4$ & $<14$ & 0.08 & $<12$ & - & - \\
\hline Zirconium 95 & $\mathrm{pCi} / \mathrm{L}$ & 29.1 & $<38$ & $<40$ & 0.57 & * & 2.4 \\
\hline Other Radionucl & ow Analy & Detectio & Limit & & & & \\
\hline Nickel 59 & $\mathrm{pCi} / \mathrm{L}$ & 340 & * 207 & $<274$ & $<340$ & - & - \\
\hline Carbon 14 & $\mathrm{pCi} / \mathrm{L}$ & 40 & 18 & 22.8 & 14.7 & 24 & 207 \\
\hline lodine 129 & $\mathrm{pCi} / \mathrm{L}$ & 2.12 & 2.3 & 1.13 & 3.79 & 2.7 & 2.8 \\
\hline Selenium 79 & $\mathrm{pCi} / \mathrm{L}$ & $<45.3$ & $<49$ & $<46$ & $<48$ & - & - \\
\hline Technicium 99 & $\mathrm{pCi} / \mathrm{L}$ & $<18.4$ & 5.9 & 3.2 & $<20$ & - & 29 \\
\hline
\end{tabular}

* Analytical result, but less than analytical Detection Limit

$A$ " $<$ " indicates that the analysis was less than that detection limit, given as a negative number

VTS=Vertical Tube Storage Basin; MB = Machine Basin; DCS = Dry Cave Section;

BKS/MPB = Open area between Bucket Storage Sectior and Monitor Pin Basin
23.0

8.7

0.22

$-$

2,800

3,500

10

18

3.5

3.9

4.9

15

7 
TABLE 4

FDD-ENG-98-0029

Page 10 of 14

1997 R-DISASSEMBLY ANALYTICAL RESULTS: LIQUID PORTION, METALS, PCB's, ANIONS

Sample Location

Sample ID

Laboratory

Lab. ID

Date Sampled

$\%$ Solids, $\mathrm{mg} / \mathrm{L}(\mathrm{ppm})$

$\mathrm{pH}$

Total Metals (ppb)

Aluminum

Arsenic

Barium

Beryllium

Boron

Cadmium

Calcium

Cesium

Chromium

Cobalt

Copper

Iron

Lead

Magnesium

Manganese

Molydenum

Nickel

Potassium

Selenium

Silver

Sodium

Strontium

Thallium

Tin

Titanium

Uranium

Vandium

Zinc

Silica

$\begin{array}{ccc}\text { VTS } & \text { MB } & \text { DCS } \\ 97293-1 & 97293-2 & 97293-3 \\ \text { GEL } & \text { GEl. } & \text { GEL } \\ 9712279-01 & 9712279-02 & 9712279-03 \\ 12 / 8 / 97 & 12 / 8 / 97 & 12 / 8 / 97 \\ & & \\ 148 & 146 & 147 \\ 8.02 & 7.74 & 6.77\end{array}$

$\mu g / L$

$\mu \mathrm{g} / \mathrm{L}$

$\mu g / L$

$\mu g / L$

$\mu g / L$

$\mu g / L$

$\mu g / L$

$\mu g / L$

$\mu g / L$

$\mu \mathrm{g} / \mathrm{L}$

$\mu \mathrm{g} / \mathrm{L}$

$\mu \mathrm{g} / \mathrm{L}$

$\mu g / L$

$\mu g / L$

$\mu \mathrm{g} / \mathrm{L}$

$\mu \mathrm{g} / \mathrm{L}$

$\mu \mathrm{g} / \mathrm{L}$

$\mu \mathrm{g} / \mathrm{L}$

$\mu g / L$

$\mu \mathrm{g} / \mathrm{L}$

$\mu g / L$

$\mu \mathrm{g} / \mathrm{L}$

$\mu g / L$

$\mu \mathrm{g} / \mathrm{L}$

$\mu \mathrm{g} / \mathrm{L}$

$\mu \mathrm{g} / \mathrm{L}$

$\mu g / L$

$\mu g / L$

$\mu g / L$

$\mu g / L$
7.74

54.9

$0.5^{\circ}$

20.4

20.4
4.68

0.02

22.7

0.07

12,700

0.29

0.97

0.073

0.84

96.1

0.05

444

1.57

0.82

0.5

28,500

94.2

0.08

14,400

154

0.05

0.43

0.82

1.86

20.1

11.2

1240
0.04

12,2010

0.27

0.82

0.04

0.76

95.15

0.015

452

0.56

0.75

0.21

27,400

92

0.05

15,000

152

0.05

0.36

0.72

1.81

20.4

11.7

1250

0.03

21.3

* 0.01

12,000

0.21

0.67

0.02

0.53

92.6

0.06

439

0.49

0.66

0.25

27,000

93.4

0.02
14,700

149

0.05

0.68

0.72

1.72

21.3

11.5

1260
BKS/MPB

97293-4

GEL

9712279-04

$12 / 8 / 97$

144

7.95
Average,

97 Liquid

GEL

$12 / 97$

146

7.62
Previous Results

\begin{tabular}{|c|c|c|}
\hline \multicolumn{3}{|c|}{ Previous Results } \\
\hline Su & & Liquid \\
\hline SRTC & CTC & CTC \\
\hline $8 / 94$ & $7 / 94$ & \\
\hline
\end{tabular}

$8 / 94 \quad 7 / 94$

$7 / 94$

7.35

Extractable Organics (ppb)

\begin{tabular}{lllllll}
\hline PCB-1016 & $\mu \mathrm{g} / \mathrm{L}$ & $<0.1$ & $<0.11$ & $<0.105$ & $<0.104$ & $<$ Det. Lim. \\
PCB-1221 & $\mu \mathrm{g} / \mathrm{L}$ & $<0.1$ & $<0.11$ & $<0.105$ & $<0.104$ & $<$ Det. Lim. \\
PCB-1232 & $\mu \mathrm{g} / \mathrm{L}$ & $<0.1$ & $<0.11$ & $<0.105$ & $<0.104$ & $<$ Det. Lim. \\
PCB-1242 & $\mu \mathrm{g} / \mathrm{L}$ & $<0.1$ & $<0.11$ & $<0.105$ & $<0.104$ & $<$ Det. Lim. \\
PCB-1248 & $\mu \mathrm{g} / \mathrm{L}$ & $<0.1$ & $<0.11$ & $<0.105$ & $<0.104$ & $<$ Det. Lim. \\
PCB-1254 & $\mu \mathrm{g} / \mathrm{L}$ & $<0.1$ & $<0.11$ & $<0.105$ & $<0.104$ & $<$ Det. Lim. \\
PCB-1260 & $\mu \mathrm{g} / \mathrm{L}$ & 0.096 & $<0.11$ & $<0.105$ & $<0.104$ & $<$ Det. Lim.
\end{tabular}

$\begin{array}{cc}45.1 & 262 \\ 0.59 & 0.63 \\ 21.1 & 21 \\ 4.68 & 7.78\end{array}$

103.7

0.60

21

5.53

* $\quad 0.02$

48

0.19

12,200

0.33

4.12

0.15

1.24

179

0.05

610

107

1.81

1.41

27,400

92.3

0.07

21,800

144

0.05

1.37

3.71

1.65

68.9

24.4

1520

*

0.030

28.9

0.08

12,275

0.28

1.65

0.07

0.84

116
$+\quad 0.06$

486

27.4

1.01

0.59

27,575

93.0

0.06

16,475

150

0.05

0.71

1.49

1.76

32.7

14.7

1318

Det. Lim.

$\begin{array}{ccc}410 & 2500 & <60 \\ - & <50 & <60 \\ <200 & <2 & <3\end{array}$

32

-

106

11

600

$<0.3$

140

12

5

$\begin{array}{ll}57 & 10 \\ 44 & <5\end{array}$

47

4

20
330

330

10

15

900

900
20

900

450

59

N.A.

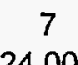

7
24,000

40

40

12,850

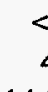

$<1$
4

14,000

70

80

$80 \quad 10,500$

$10,500 \quad 53$

Anions (ppm)

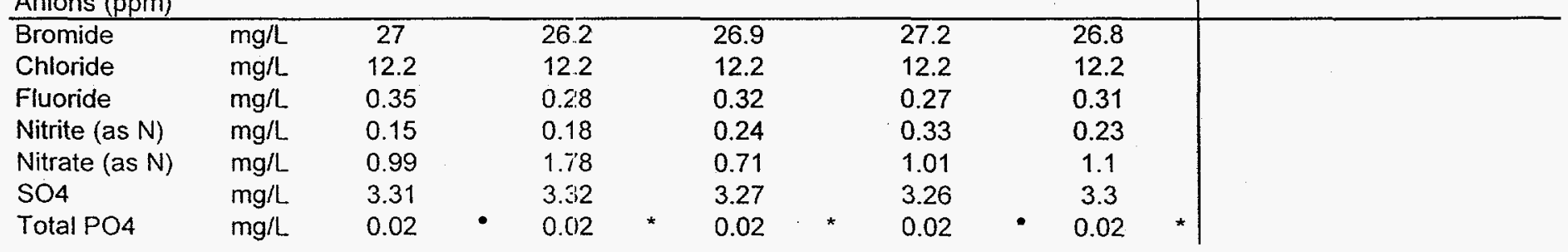

$V T S=V e r t i c a l$ Tube Storage Basin; $M B=$ Machine Basin; DCS = Dry Cave Section;

BKS/MPB = Open area between Bucket Storage Section and Monitor Pin Basin

* = Less than anlytical Detection Limit, N. A. = Not analyzed 
LIQUID PORTION - RADIONUCLIDES

Sample Location

Sample ID

Laboratory

Lab. ID

Date Sampled

Gross Alpha
Non-volatile Gross Beta $\mathrm{pCi} / \mathrm{L}$

\begin{tabular}{ccccc|c} 
VTS & IVB & DCS & BKS/MPB & Average & Previous Results \\
97293-1 & $97293-2$ & $97293-3$ & $97293-4$ & Liquid & Supernate
\end{tabular}

GEL GEL GEL GEL

9712279-05 9712279-06 $97122791-07 \quad 97122791-08$

$12 / 8 / 97 \quad 12 / 8 / 97 \quad 12 / 8 / 97 \quad 12 / 8 / 97$

\begin{tabular}{|c|c|c|}
\hline & & \\
\hline Liquid & Supernate & Liquid \\
\hline GEL & SRTC & SRTC \\
\hline $12 / 97$ & $7 / 94$ & $12 / 96$ \\
\hline
\end{tabular}

$80,000 \quad 110,000$

23,500

38,075

207

29

171

6,125

Alpha Spectroscopy, Nuclides Detected

pCir

36,100

$92, \overline{900}$

92,500

93,000

92,800

Strontium 90

$\mathrm{pCi} / \mathrm{L}$

$\mathrm{pCi} / \mathrm{m}$

18,800

$23, \overline{100}$

38,600

26,800

246

191

159

233
$<24.1$

24.9

30.9

30.1

Alpha Spectroscopy, Nuclides Below Analytical Detection Limit

\begin{tabular}{|c|c|c|c|c|c|c|c|c|c|}
\hline $\mathrm{U}-235$ & $\mathrm{pCi} / \mathrm{L}$ & 1.54 & 6 & 4.47 & $\bar{\star}$ & 14.4 & $\bar{k}$ & 19.7 & * \\
\hline$U-238$ & $\mathrm{pCi} / \mathrm{L}$ & 3.07 & • & $<16.4$ & & 6.38 & - & 16.6 & * \\
\hline Np-237 & $\mathrm{pCi} / \mathrm{L}$ & 0.48 & * & 0.54 & * & 0.37 & * & 0.74 & \\
\hline Pu-238 & $\mathrm{pCi} / \mathrm{L}$ & $<0.86$ & & $\because 1.1$ & & $<0.91$ & & $<1.1$ & \\
\hline Pu-239/240 & $\mathrm{pCi} / \mathrm{L}$ & 0.14 & • & 0.11 & * & 0.23 & - & 0.41 & * \\
\hline $\mathrm{Pu}-241$ & $\mathrm{pCi} / \mathrm{L}$ & $<113$ & & $<: 126$ & & $<137$ & & 6.19 & * \\
\hline Pu-242 & $\mathrm{pCi} / \mathrm{L}$ & 0.12 & * & 0.08 & * & 0.27 & - & 0.24 & * \\
\hline Am-241 & $\mathrm{pCi} / \mathrm{L}$ & $<67.1$ & & $<: 105$ & & 40.8 & * & $<119$ & \\
\hline
\end{tabular}

Gamma PHA Scan - Radionuclides Below Analytical Detection Limit

\begin{tabular}{|c|c|c|c|c|c|c|c|c|}
\hline Actinium 228 & pCill & 2.22 & 6.14 & $\bullet$ & 5.19 & $\bullet$ & 0.61 & 3.5 \\
\hline Antimony 124 & pCill & $<13.8$ & $<33$ & & 12.2 & * & 12.8 & * \\
\hline Antimony 125 & $\mathrm{pCi} / \mathrm{L}$ & 7.67 & 26.2 & • & $<163$ & & $<168$ & \\
\hline Barium 133 & $\mathrm{pCi} / \mathrm{L}$ & 4.96 & $<58.2$ & & 5 & * & 1.82 & 3.9 \\
\hline Californium 249 & $\mathrm{pCi} / \mathrm{L}$ & - & - & & - & & - & * \\
\hline Californium 251 & $\mathrm{pCi} / \mathrm{L}$ & - & - & & - & & - & \\
\hline Cerium 141 & $\mathrm{pCi} / \mathrm{L}$ & 4.16 & 18.3 & * & 22.1 & * & $<97$ & 14.9 \\
\hline Cerium 144 & $\mathrm{pCi} / \mathrm{L}$ & $<89$ & 47.3 & $\star$ & $<279$ & & 109 & * \\
\hline Cesium- 134 & $\mathrm{pCi} / \mathrm{L}$ & $<11$ & 1.02 & * & 3.1 & * & $<33$ & \\
\hline Cesium 135 & $\mathrm{pCi} / \mathrm{L}$ & 7.2 & 52.2 & * & 175 & * & $<214$ & \\
\hline Cobalt 57 & $\mathrm{pCi} / \mathrm{L}$ & $<11$ & $<28$ & & 18.5 & * & $<38$ & \\
\hline Cobalt 58 & $\mathrm{pCi} / \mathrm{L}$ & $<2.5$ & 2.02 & * & 2.1 & * & 2.29 & 0.15 \\
\hline Cobalt 60 & $\mathrm{pCi} / \mathrm{L}$ & 0.48 & 4.94 & * & 1.81 & * & 3.51 & 2.7 \\
\hline Europium 152 & $\mathrm{pCi} / \mathrm{L}$ & $<49$ & 56.3 & * & 66.4 & * & $<144$ & \\
\hline Europium 154 & pCilL & 1.5 & $<11$ & & $<11$ & & $<11$ & \\
\hline Europium 155 & $\mathrm{pCi} / \mathrm{L}$ & $<44$ & $<102$ & & 20.5 & * & $<155$ & \\
\hline Lead 212 & $\mathrm{pCi} / \mathrm{L}$ & 6.76 & 7.93 & $*$ & $<74$ & & 13.6 & 9.4 \\
\hline Manganese 54 & $p C i / L$ & $<2.1$ & 4.16 & * & $<5.4$ & - & 1.52 & $\star$ \\
\hline Neptunium 239 & $\mathrm{pCi} / \mathrm{L}$ & 34.1 & 109 & * & $<267$ & & $<283$ & \\
\hline Niobium 94 & $p C i / L$ & 0.16 & $<5.8$ & & $<5.9$ & & 0.98 & * \\
\hline Potasium 40 & $\mathrm{pCi} / \mathrm{L}$ & 16 & 88.1 & & 20.1 & * & 69.6 & 48 \\
\hline Promethium 144 & $\mathrm{pCi} / \mathrm{L}$ & 0.36 & $<5.9$ & & 0.13 & * & 2.34 & * \\
\hline Promethium 146 & $\mathrm{pCi} / \mathrm{L}$ & 5.04 & $<79$ & & $<85$ & & 44.4 & * \\
\hline Ruthenium 103 . & $\mathrm{pCi} / \mathrm{L}$ & $<23$ & $<61$ & & 16.1 & * & $<70$ & \\
\hline Ruthenium 106 & $\mathrm{pCi} / \mathrm{L}$ & 27.6 & 54.2 & * & 27.8 & * & $<328$ & 37 \\
\hline Sodium 22 & $\mathrm{pCi} / \mathrm{L}$ & 0.55 & $<3.8$ & & $<4.1$ & & $<3.9$ & \\
\hline $\operatorname{Tin} 113$ & $\mathrm{pCi} / \mathrm{L}$ & 14.2 & 28.6 & * & $<73$ & & $<75$ & \\
\hline $\operatorname{Tin} 126$ & $\mathrm{pCi} / \mathrm{L}$ & 0.31 & 0.88 & $\bullet$ & 3.12 & * & 1.8 & 1.5 \\
\hline Yttrium 88 & $\mathrm{pCi} / \mathrm{L}$ & 0.59 & $<3.7$ & & 0.42 & * & 1.35 & 0.8 \\
\hline Zinc 65 & $\mathrm{pCi} / \mathrm{L}$ & $<4.3$ & $<9.1$ & & 5.71 & * & $<9.1$ & $\cdot$ \\
\hline Zirconium 95 & $\mathrm{pCi} / \mathrm{L}$ & 1.25 & 5.86 & * & $<12.1$ & & 0.023 & 2.4 \\
\hline \multicolumn{9}{|c|}{ Other Radionuclides Below Analytical Detection Limit } \\
\hline Nickel 59 & $\mathrm{pCi} / \mathrm{L}$ & $<469$ & 381 & $\bullet$ & $<355$ & & 83.7 & * \\
\hline lodine 129 & pCill & $<3.04$ & 6.77 & & 0.47 & * & 1.27 & 2.8 \\
\hline Selenium 79 & $\mathrm{pCi} / \mathrm{L}$ & 2.55 & $<47$ & & $<45$ & & $<47$ & \\
\hline
\end{tabular}

- Analytical result reported, but less than analytical Detection Limit, and the Reporting Limit

$A$ " $<$ " indicates that the analysis was less than that detection limit; reported as a negative number

VTS=Vertical Tube Storage Basin; MB = Machine Basin; DCS = Dry Cave Section;

BKS/MPB = Open area between Bucket Storage Section and Monitor Pin Basin 
TABLE 6

1997 R-DISASSEMBLY ANALYTICAL RESULTS: SEDIMENT PORTION; METALS

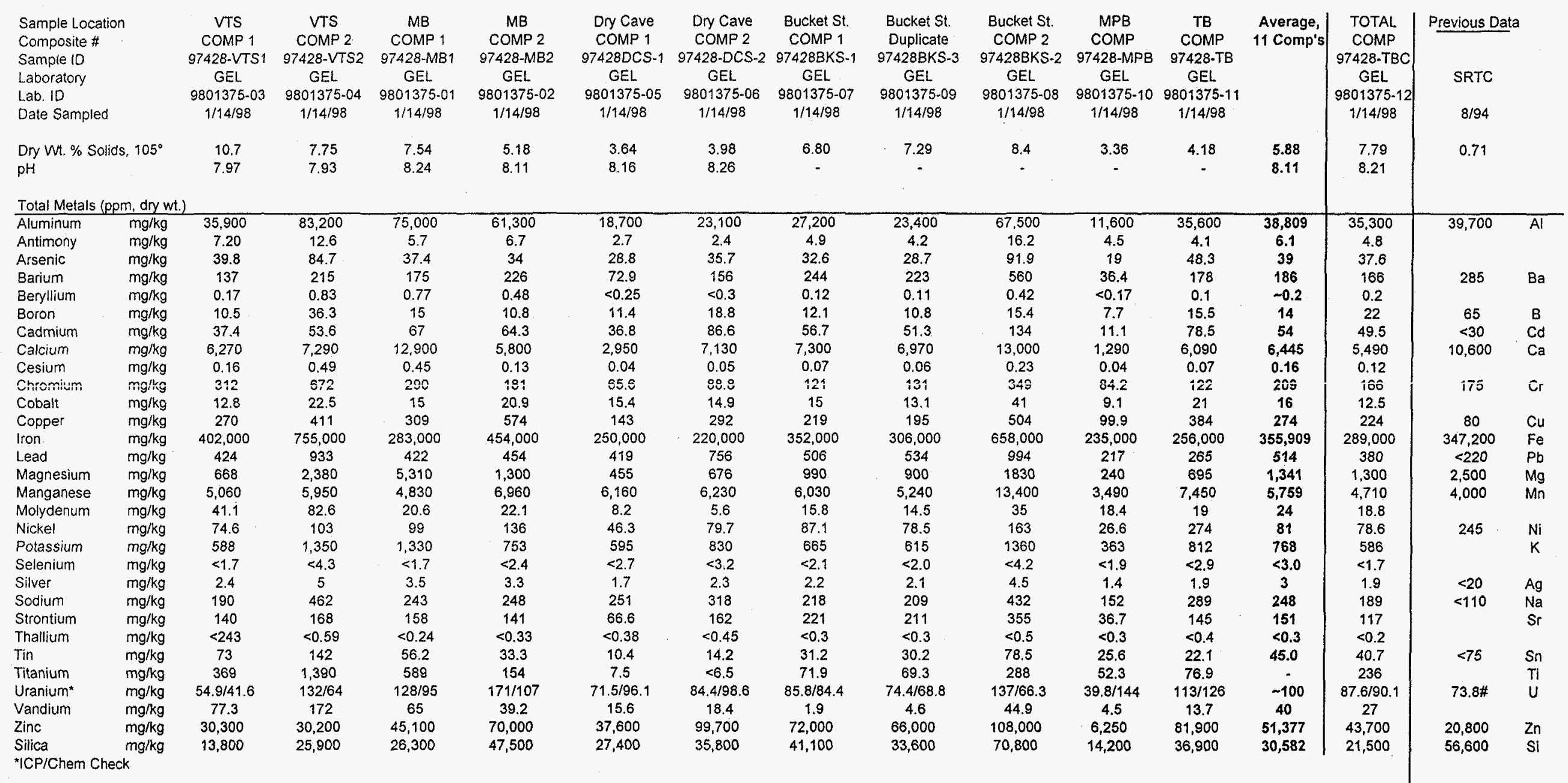

$\begin{array}{lllllllll}\text { Total PO4 } & \mathrm{mg} / \mathrm{kg} & 7.95 & 19.8 & 3.3 & 19.8 & 8.3 & 8.8 & 4.2\end{array}$

12.7

10.3

5.7

$9.1 \mid 5.3$

\# Note, 90 micrograms U/gm solid $\times 10-6 \mathrm{gms} U /$ microgm U $\times 3.4 \times 10-7 \mathrm{Ci} / \mathrm{gm} \mathrm{U}=300 \times 10-13 \mathrm{Ci} / \mathrm{gm} \mathrm{solid}=30 \mathrm{pCi} / \mathrm{gm}$ 
TABLE 7

FDD-ENG-98-0029*

Page 13 of 14

1997 R-DISASSEMBLY ANALYTICAL RESULTS: SEDIMENT PORTION; PCB'S, PESTICIDES, \& ANIONS

\begin{tabular}{|c|c|c|c|c|c|c|c|c|c|c|c|c|c|c|c|}
\hline \multicolumn{2}{|l|}{ Sample Location } & VTS & VTS & MB & MB & Dry Cave & Dry Cave & Bucket St. & Bucket St. & Bucket St. & MPB & TB & Average, & TOTAL & $\begin{array}{l}\text { Previous } \\
\text { Poculte }\end{array}$ \\
\hline \multirow{2}{*}{\multicolumn{2}{|c|}{$\begin{array}{l}\text { Composite \# } \\
\text { Sample ID }\end{array}$}} & COMP 1 & COMP 2 & COMP 1 & COMP 2 & COMP 1 & COMP 2 & COMP 1 & Duplicate & COMP 2 & COMP & $\begin{array}{l}\text { COMP } \\
97428-T B\end{array}$ & & $\begin{array}{c}\text { COMP } \\
\text { 97428-TCB }\end{array}$ & Results \\
\hline \multirow{2}{*}{\multicolumn{2}{|c|}{$\begin{array}{l}\text { Sample ID } \\
\text { Laboratory }\end{array}$}} & 97428-VTS1 & $97428-V T S 2$ & 97428-MB1 & 97428-MB2 & 97428DCS-1 & 97428-DCS-2 & 97428 BKS -1 & $97428 \mathrm{BKS}-3$ & & 97428-MPB & & & & \\
\hline & & GEL & GEL & GEL & GEL & GEL & GEL & GEL & GEL & GEL & GEL & GEL & & GEL & CTC \\
\hline \multicolumn{2}{|l|}{ Lab. ID } & $9801375-03$ & $9801375-04$ & $9801375-01$ & $9801375-02$ & $9801375-05$ & $9801375-06$ & $9801375-07$ & $9801375-09$ & $9801375-08$ & $9801375-10$ & $9801375-11$ & & $9801375-12$ & \\
\hline \multicolumn{2}{|l|}{ Date Sampled } & $1 / 14 / 98$ & $1 / 14 / 98$ & $1 / 14 / 98$ & 1/14/98 & $1 / 14 / 98$ & $1 / 14 / 98$ & $1 / 14 / 98$ & $1 / 14 / 98$ & $1 / 14 / 98$ & $1 / 14 / 98$ & $1 / 14 / 98$ & & $1 / 14 / 98$ & $7 / 94$ \\
\hline & 10.7 & 7.75 & 7.54 & 5.18 & 3.64 & 3.98 & 6.80 & 7.29 & 8.40 & 3.36 & 4.18 & 6.18 & 7.79 & \\
\hline \multicolumn{2}{|c|}{ pH } & 7.97 & 7.93 & 8.24 & 8.11 & 8.16 & 8.26 & 8.50 & 8.50 & 8.22 & 8.03 & 8.90 & 8.18 & 8.21 & \\
\hline \multicolumn{16}{|c|}{ PCB's (mg $/ \mathrm{kg}$, dry wt, ppm) } \\
\hline PCB-1016 & $\mathrm{mg} / \mathrm{kg}$ & $<0.13$ & $<0.34$ & $<0.25$ & $<0.18$ & $<0.11$ & $<0.13$ & $<0.32$ & $<0.17$ & $<0.33$ & $<0.07$ & $<0.55$ & - & $<0.13$ & - \\
\hline PCB-1221 & $\mathrm{mg} / \mathrm{kg}$ & $<0.13$ & $<0.34$ & $<0.25$ & $<0.18$ & $<0.11$ & $<0.13$ & $<0.32$ & $<0.17$ & $<0.33$ & $<0.07$ & $<0.55$ & - & $<0.13$ & - \\
\hline PCB-1232 & $\mathrm{mg} / \mathrm{kg}$ & $<0.13$ & $<0.34$ & $<0.25$ & $<0.18$ & $<0.11$ & $<0.13$ & $<0.32$ & $<0.17$ & $<0.33$ & $<0.07$ & $<0.55$ & - & $<0.13$ & - \\
\hline PCB 1242 & $\mathrm{mg} / \mathrm{kg}$ & $<0.13$ & $<0.34$ & $<0.25$ & $<0.18$ & $<0.11$ & $<0.13$ & $<0.32$ & $<0.17$ & $<0.33$ & $<0.07$ & $<0.55$ & - & $<0.13$ & - \\
\hline PCB-1248 & $\mathrm{mg} / \mathrm{kg}$ & $<0.13$ & $<0.34$ & $<0.25$ & $<0.18$ & $<0.11$ & $<0.13$ & $<0.32$ & $<0.17$ & $<0.33$ & $<0.07$ & $<0.55$ & - & $<0.13$ & • \\
\hline PCB.1254 & $\mathrm{mg} / \mathrm{kg}$ & 0.88 & 2.75 & 1.71 & 1.53 & 0.92 & 0.62 & 2.26 & 2.18 & 2.57 & 0.91 & 5.12 & 1.87 & 1.00 & $15 \& 99$ \\
\hline PCB. 1260 & $\mathrm{mg} / \mathrm{kg}$ & $<0.13$ & $<0.34$ & $<0.25$ & $<0.18$ & $<0.11$ & $<0.13$ & $<0.32$ & $<0.17$ & $<0.33$ & $<0.07$ & $<0.55$ & - & $<0.13$ & • \\
\hline Pesticides, $\mathrm{mg} / \mathrm{kg}$ & $\mathrm{mg} / \mathrm{kg}$ & & & & & & & & & & & & & & \\
\hline $4,4^{\prime}$-DDD & $\mathrm{mg} / \mathrm{kg}$ & $<0.025$ & $<0.067$ & $<0.051$ & $<0.037$ & $<0.022$ & $<0.026$ & $<0.064$ & $<0.034$ & $<0.065$ & $<0.015$ & $<0.11$ & - & $<0.026$ & \\
\hline 4,4-DDE & $\mathrm{mg} / \mathrm{kg}$ & $<0.025$ & $<0.067$ & $<0.051$ & $<0.037$ & $<0.022$ & $<0.026$ & $<0.064$ & $<0.034$ & $<0.065$ & $<0.015$ & $<0.11$ & - & $<0.026$ & \\
\hline 4.4'-DOT & $\mathrm{mg} / \mathrm{kg}$ & $<0.025$ & $<0.067$ & $<0.051$ & $<0.037$ & $<0.022$ & $<0.026$ & $<0.064$ & $<0.034$ & $<0.065$ & $<0.015$ & $<0.11$ & - & $<0.026$ & \\
\hline Aidirin & $\mathrm{mg} / \mathrm{kg}$ & $<0.025$ & $<0.067$ & $<0.051$ & $<0.037$ & $<0.022$ & $<0.026$ & $<0.064$ & $<0.034$ & $<0.065$ & $<0.015$ & $<0.11$ & - & $<0.026$ & \\
\hline Dieldrin & $\mathrm{mg} / \mathrm{kg}$ & $<0.025$ & $<0.067$ & $<0.051$ & $<0.037$ & $<0.022$ & $<0.026$ & $<0.064$ & $<0.034$ & $<0.065$ & $<0.015$ & $<0.11$ & - & $<0.026$ & \\
\hline Endosulfan I & $\mathrm{mg} / \mathrm{kg}$ & $<0.025$ & $<0.067$ & $<0.051$ & $<0.037$ & $<0.022$ & $<0.026$ & $<0.064$ & $<0.034$ & $<0.065$ & $<0.015$ & $<0.11$ & - & $<0.026$ & \\
\hline Endosufan $\|$ & $\mathrm{mg} / \mathrm{kg}$ & $<0.025$ & $<0.067$ & $<0.051$ & $<0.037$ & $<0.022$ & $<0.026$ & $<0.064$ & $<0.034$ & $<0.065$ & $<0.015$ & $<0.11$ & - & $<0.026$ & . \\
\hline Endosulfan sulfate & $\mathrm{mg} / \mathrm{kg}$ & $<0.025$ & $<0.067$ & $<0.051$ & $<0.037$ & $<0.022$ & $<0.026$ & $<0.064$ & $<0.034$ & $<0.065$ & $<0.015$ & $<0.11$ & - & $<0.026$ & \\
\hline Heptachlor & $\mathrm{mg} / \mathrm{kg}$ & $<0.025$ & $<0.067$ & $<0.051$ & $<0.037$ & $<0.022$ & $<0.026$ & $<0.064$ & $<0.034$ & $<0.065$ & $<0.015$ & $<0.11$ & - & $<0.026$ & \\
\hline Heptachlor epoxide & $\mathrm{mg} / \mathrm{kg}$ & $<0.025$ & $<0.067$ & $<0.051$ & $<0.037$ & $<0.022$ & $<0.026$ & $<0.064$ & $<0.034$ & $<0.065$ & $<0.015$ & $<0.11$ & - & $<0.026$ & \\
\hline Methoxychlor & $\mathrm{mg} / \mathrm{kg}$ & $<0.025$ & $<0.067$ & $<0.051$ & $<0,037$ & $<0.022$ & $<0.026$ & $<0.064$ & $<0.034$ & $<0.065$ & $<0.015$ & $<0.11$ & - & $<0.026$ & \\
\hline Toxaphene & $\mathrm{mg} / \mathrm{kg}$ & $<0.63$ & $<1.68$ & $<1.27$ & $<0.92$ & $<0.55$ & $<0.66$ & $<1.60$ & $<0.84$ & $<1.63$ & $<0.36$ & $<2.78$ & - & $<0.65$ & \\
\hline Alpha-BHC & $\mathrm{mg} / \mathrm{kg}$ & $<0.013$ & $<0.034$ & $<0.025$ & $<0.018$ & $<0.011$ & $<0.013$ & $<0.032$ & $<0.017$ & $<0.033$ & $<0.007$ & $<0.055$ & - & $<0.013$ & \\
\hline Alpha-Chiordane & $\mathrm{mg} / \mathrm{kg}$ & $<0.013$ & $<0.034$ & $<0.025$ & $<0.018$ & $<0.011$ & $<0.013$ & $<0.032$ & $<0.017$ & $<0.033$ & $<0.007$ & $<0.055$ & - & $<0.013$ & \\
\hline Beta-BHC & $\mathrm{mg} / \mathrm{kg}$ & $<0.013$ & $<0.034$ & $<0.025$ & $<0,018$ & $<0.011$ & $<0.013$ & $<0.032$ & $<0.017$ & $<0.033$ & $<0.007$ & $<0.055$ & - & $<0.013$ & \\
\hline Delta-BHC & $\mathrm{mg} / \mathrm{kg}$ & $<0.013$ & $<0.034$ & $<0.025$ & $<0.018$ & $<0.011$ & $<0.013$ & $<0.032$ & $<0.017$ & $<0.033$ & $<0.007$ & $<0.055$ - & - & $<0.013$ & \\
\hline Gamma.BHC & $\mathrm{mg} / \mathrm{kg}$ & $<0.013$ & $<0.034$ & $<0.025$ & $<0.018$ & $<0.011$ & $<0.013$ & $<0.032$ & $<0.017$ & $<0.033$ & $<0.007$ & $<0.055$ & - & $<0.013$ & \\
\hline gamma-chlordane & $\mathrm{mg} / \mathrm{kg}$ & $<0.013$ & $<0.034$ & $<0.025$ & $<0.018$ & $<0.011$ & $<0.013$ & $<0.032$ & $<0.017$ & $<0.033$ & $<0.007$ & $<0.055$ & - & $<0.013$ & \\
\hline
\end{tabular}

\begin{tabular}{|c|c|c|c|c|c|c|c|c|c|c|c|c|c|c|}
\hline Bromide & $\mathrm{mg} / \mathrm{kg}$ & & & & & & & & & & & & & 228 \\
\hline Chloride & $\mathrm{mg} / \mathrm{kg}$ & & & & & & & & & & & & & 102 \\
\hline Fluoride & $\mathrm{mg} / \mathrm{kg}$ & & & & & & & & & & & & & 4.6 \\
\hline Nitrite (as N) & $\mathrm{mg} / \mathrm{kg}$ & & & & & & & & & & & & & $<3.0$ \\
\hline Nitrate (as N) & $\mathrm{mg} / \mathrm{kg}$ & & & & & & & & & & & & & 7.1 \\
\hline $\mathrm{SO} 4$ & $\mathrm{mg} / \mathrm{kg}$ & & & & & & & & & & & & & 106 \\
\hline Total PO4 & $\mathrm{mg} / \mathrm{kg}$ & 7950 & 19800 & 3300 & 1810 & 8320 & 8870 & 4170 & 4820 & 12700 & 1030 & 5730 & 6,615 & 5320 \\
\hline
\end{tabular}

VTS=Vertical Tube Storage Area; MB = Machine Basin; DCS = Dry Cave Section; MPB = Monitor Pin Basin; TB = Transfer Bay 
TABLE 8

Sample Location

Sample 1D

Laboratory

Lab. ID

Date Sampled
Total Basin Composite (FBC)

97428-TCB

GEL

9801375-13

$1 / 14 / 98$

Analyses reported as pCi/gm, dry wt.

Gross Alpha

pCi/gm

Alpha Spectroscopy, Nuclides

U-233/234

U-235

U-238

$\mathrm{Np}-237$

Pu-238

Pu-239/240

Pu-241

Pu-242

Arn-241

$\mathrm{pCi} / \mathrm{gm}$

pCi/gm

pCilgm

pCi/gm

pCilgm

pCi/gm

pCi/gm

pCi/gm

pCilgm

Previous Results

Gamma PHA Scan - Radionuclides

\begin{tabular}{|c|c|c|c|c|c|c|}
\hline Actinium 228 & pCi/gm & < Det. Lim. & 0.004 & & & \\
\hline Antimony 124 & pCi/gm & 2.0 & - & & & \\
\hline Antimony 125 & $\mathrm{pCi} / \mathrm{gm}$ & 12 & $\star$ & & & \\
\hline Barium 133 & $\mathrm{pCi} / \mathrm{gm}$ & < Det. Lim. & 0.004 & & & \\
\hline Californium 249 & pCi/gm & 4.4 & * & & & \\
\hline Californium 251 & $\mathrm{pCi} / \mathrm{gm}$ & 3.6 & - & & & \\
\hline Cerium 141 & $\mathrm{pCi} / \mathrm{gm}$ & 14 & 0.015 & & & . \\
\hline Cerium 144 & $\mathrm{pCi} / \mathrm{gm}$ & $<$ Det Lim. & - & & & \\
\hline Cesium 134 & $\mathrm{pCi} / \mathrm{gm}$ & < Det. Lim. & - & & & \\
\hline Cesium 135 & $\mathrm{pCi} / \mathrm{gm}$ & 6.4 & * & & & \\
\hline Cesium 137 & pCilgm & 20,500 & 92 & 2,550 & 80 & 110 \\
\hline Cobalt 57 & $\mathrm{pCi} / \mathrm{gm}$ & 4.0 & - & & & \\
\hline Cobalt 58 & $\mathrm{pCi} / \mathrm{gm}$ & $<$ Det. Lim. & 0.0002 & & & \\
\hline Cobalt 60 & $\mathrm{pCi} / \mathrm{gm}$ & 47,900 & 0.003 & 1,760 & N.D. & N.D. \\
\hline Europium 152 & pCi/gm & 46 & - & & & \\
\hline Europium 154 & $\mathrm{pCi} / \mathrm{gm}$ & 367 & - & & & \\
\hline Europium 155 & pCi/gm & 44 & - & & & \\
\hline Lead 212 & pCilgm & 3.5 & 0.009 & & & \\
\hline Manganese 54 & $\mathrm{pCi} / \mathrm{gm}$ & 7.3 & - & & & \\
\hline Neptunium 239 & $\mathrm{pCi} / \mathrm{gm}$ & 14 & * & & & \\
\hline Niobium 94 & pCi/gm & 6.58 & * & & & \\
\hline Potasium 40 & $\mathrm{pCi} / \mathrm{gm}$ & < Det. Lim. & 0.048 & & & \\
\hline Promethium 144 & $\mathrm{pCi} / \mathrm{gm}$ & < Det. Lim. & - & & & \\
\hline Promethium 146 & pCi/gm & < Det. Lim. & - & & & \\
\hline Ruthenium 103 & $\mathrm{pCi} / \mathrm{gm}$ & < Det. Lim. & - & & & \\
\hline Ruthenium 106 & $\mathrm{pCi} / \mathrm{gm}$ & 0.71 & 0.037 & & & \\
\hline Sodium 22 & $\mathrm{pCi} / \mathrm{gm}$ & 135 & - & & & \\
\hline Tin 113 & pCilgm & < Det. Lim. & - & & & \\
\hline $\operatorname{Tin} 126$ & $\mathrm{pCi} / \mathrm{gm}$ & < Det. Lim. & 0.002 & & & \\
\hline Yttrium 88 & $\mathrm{pCi} / \mathrm{gm}$ & 41 & 0.0008 & & & \\
\hline Zinc 65 & $\mathrm{pCi} / \mathrm{gm}$ & 78 & - & & & \\
\hline Zirconium 95 & $\mathrm{pCi} / \mathrm{gm}$ & 46 & 0.002 & & & \\
\hline \multicolumn{7}{|c|}{ Other Radionuclides } \\
\hline Nickel 59 & $\mathrm{pCi} / \mathrm{gm}$ & 287 & - & & & \\
\hline Strontium-90 & $\mathrm{pCi} / \mathrm{gm}$ & 3,160 & 23.5 & N.A. & N.A. & N.A. \\
\hline Tritium & pCilgm & 397,000 & 38,075 & 345,000 & 41,000 & 39,000 \\
\hline Carbon 14 & $\mathrm{pCi} / \mathrm{gm}$ & 6,170 & 0.21 & & & \\
\hline lodine 129 & $\mathrm{pCi} / \mathrm{gm}$ & 11 & 0.003 & & & \\
\hline Selenium 79 & $\mathrm{pCi} / \mathrm{gm}$ & < Det. Lim. & - & & & \\
\hline Technicium 99 & pCi/gm & 25 & 0.029 & & & \\
\hline
\end{tabular}

- Analytical result reported, but it was less than analytical detection Limit

A "< Det. Lim." indicates that the analysis was less than the detection limit, given as a negative number

The Total Basin Composite sample was collected from 9 separate locations throughout the basin;

the residues (after PCB samples were taken) from the other 11 composite samples were also added to the TBC

@ "TRU limit for Pu-241 is 3,500,000 pCi/gm (3500 nCilgm).

\# $30 \mathrm{pCi} / \mathrm{gm}$ based on GEL's ICP analyses vs. alpha spectroscopy; \# is based on SRTC's ICP-MS result

N. D. = Not Detected; $N . A_{1}=$ not Analyzed 